\title{
Long non-coding RNA HCP5 promotes prostate cancer cell proliferation by acting as the sponge of miR-4656 to modulate CEMIP expression
}

\author{
RENGUANG HU and ZHONGJIE LU \\ Department of Urology, The People's Hospital of Hanchuan City, Hanchuan, Hubei 431600, P.R. China
}

Received February 11, 2019; Accepted September 4, 2019

DOI: $10.3892 /$ or.2019.7404

\begin{abstract}
Aberrant expression of long noncoding RNAs (lncRNAs) has been demonstrated in human cancers and regulates the malignant behavior of cancer cells. Previous studies demonstrated the critical involvement of lncRNA histocompatibility leukocyte antigen (HLA) complex P5 (HCP5) in the development of cancers, however, the function of HCP5 in prostate cancer has not been reported. In the present study, we found the overexpressed expression of HCP5 in prostate cancer tissues and cell lines via RT-qPCR analysis. High expression of HCP5 was positively correlated with the metastasis of prostate cancer. Downregulation of HCP5 inhibited the proliferation, colony formation and induced apoptosis of prostate cancer cells. Functional experiments demonstrated that HCP5 acted as a competing endogenous RNA (ceRNA) to sponge miR-4656. Ectopic expression of HCP5 decreased the expression of miR-4656 in prostate cancer cells. MiR-4656 was found to be decreased in prostate cancer tissues and was negatively correlated with the expression of HCP5. Further luciferase reporter assay revealed that miR-4656 was able to bind the 3'-untranslated region (3'-UTR) of the cell migration inducing hyaluronidase 1 (CEMIP) and suppressed the expression of CEMIP. Consistent with the negative regulation of miR-4656 by HCP5, western blot analysis uncovered that overexpression of HCP5 upregulated the abundance of CEMIP in prostate cancer cells. The CCK-8 assay showed that depletion of CEMIP significantly inhibited the HCP5-promoted proliferation of prostate cancer cells. Collectively, our data provide a novel mechanism by which $\mathrm{HCP} 5$ regulates the progression of prostate cancer.
\end{abstract}

Correspondence to: Dr Zhongjie Lu, Department of Urology, The People's Hospital of Hanchuan City, 1 Avenue of Han Chuan City, Hanchuan, Hubei 431600, P.R. China

E-mail: zhigangdoc@163.com

Key words: prostate cancer, HCP5, miR-4656, CEMIP

\section{Introduction}

Prostate cancer is the most commonly diagnosed cancer in males and is also the leading cause of cancer-associated mortality among males $(1,2)$. Even though remarkable progress has been made in the treatment of prostate cancer, most patients are diagnosed with middle- or late-stage disease, which leads to disease progression and a poor prognosis (3). Currently, androgen-deprivation therapy is widely used in the treatment of prostate cancer (4-6); however, many patients develop drug resistance which contributes to a high male mortality rate. Therefore, there is an urgent need to identify novel regulators involved in modulating the progression of prostate cancer and to design more effective therapeutic strategies.

Long non-coding RNAs (lncRNAs) are a class of RNAs processing more than 200 nucleotides without protein-coding capacity (7). Increasing evidence suggests that lncRNAs play important roles in a variety of biological processes via diverse mechanisms, including chromatin regulation, alternative splicing and epigenetic control (8-10). Notably, previous studies have uncovered that lncRNAs act as competing endogenous RNA (ceRNAs) to sponge the function of microRNAs (miRNAs) $(11,12)$. miRNAs are characterized as a group of small ( 18-22 nt), single-stranded, non-coding RNAs $(13,14)$. It is well documented that miRNAs negatively regulate the gene expression via binding the 3'-untranslated region (3'-UTR) of target mRNAs, which results in the degradation or translation inhibition of mRNAs $(15,16)$. As important regulators of gene expression, both lncRNAs and miRNAs are widely involved in tumorigenesis (17-19). For example, lncRNA PVT1 was found to function as a ceRNA to regulate the expression of HIFl $\alpha$ via sponging miR-186 in gastric cancer (20). In prostate cancer, lncRNA HOTTIP was found to promote the proliferation and migration of prostate caner cells via sponging miR-216a-5p (21). Additionally, lncRNA RNCR3 was demonstrated to enhance the progression of prostate cancer by targeting miR-185-5p (22). These results highlight the critical roles of lncRNAs in regulating the growth of cancer cells by acting as ceRNAs.

IncRNA histocompatibility leukocyte antigen (HLA) complex P5 (HCP5) is primarily detected in immune cells, including spleen, blood and thymus (23). In addition to its function in autoimmunity, aberrant expression of HCP5 has been found in human cancers (24). A recent study reported 
that HCP5 is upregulated in glioma tissues (25). HCP5 was found to modulate the proliferation, migration and invasion of glioma cells via binding to miR-139 and to increase the expression of Runt1 (25). Additionally, HCP5 was found to promote the progression of cervical cancer by regulating MACC1 by suppressing miR-15a (24). The HCP5 region was also identified as the susceptibility locus for HCV-related hepatocellular carcinoma (26). However, the function of HCP5 in prostate cancer remains uncharacterized.

In the present study, HCP5 was found to be highly expressed in prostate cancer. Mechanistically, HCP5 was found to promote the proliferation of prostate cancer cells via sponging miR-4656 to upregulate the expression of CEMIP. Our results provide novel insight into clarifying the critical function of HCP5 in regulating the progression of prostate cancer.

\section{Materials and methods}

Tissue samples. Fifty paired prostate cancer tissues and corresponding normal tissues were collected from patients (47-78 years of age) who were diagnosed with prostate cancer and underwent surgery at The People's Hospital of Hanchuan City from August 2012 to May 2014. The samples were reviewed by three pathologists independently. None of the patients were administered local or systemic treatment prior to surgery. Informed consent was provided by all of the enrolled patients, and the study was approved by the Ethics Committee of The People's Hospital of Hanchuan City (accession no. LL2017014).

Cells and transfection. The prostate cancer cell lines PC3, Du145, PPC-1 and C4-2 were purchased from the Institute of Biochemistry (Shanghai, China). The PPC-1 cell line was authenticated with the STR profile. Cells were cultured in RPMI-1640 medium (Gibco; Thermo Fisher Scientific, Inc.) supplemented with $10 \%$ fetal bovine serum (FBS; Gibco; Thermo Fisher Scientific, Inc.), $100 \mathrm{mg} / \mathrm{ml}$ streptomycin and $200 \mathrm{U} / \mathrm{ml}$ penicillin. The nontumorigenic human prostate epithelial cell line 9 (NHP9) was maintained in PrEBM medium (Clonetics) containing insulin, hydrocortisone, epidermal growth factor and bovine pituitary extract. Cells were maintained in an incubator at $37^{\circ} \mathrm{C}$ with $5 \% \mathrm{CO}_{2}$. For the overexpression of HCP5, the full-length of HCP5 was constructed into the pcDNA vector. miR-4656 mimics (UGGGCUGAGGGCAGGAGGCCUGU; HMI1647) and control-miRNA (GGUUCGUACGUACACUGUUCA; HMC0002) (both from Sigma-Aldrich; Merck KGaA) were transfected $(10,000$ cells) at the concentration of $25 \mu \mathrm{M}$ with Lipofectamine 2000 (Invitrogen; Thermo Fisher Scientific, Inc.) for $15 \mathrm{~min}$ at room temperature (RT). After transfection for $48 \mathrm{~h}$, the cells were harvested for further analysis. shRNA-HCP5 $(0.5 \mu \mathrm{g})$ (CCGGGATCTATTACC TGTGCCTGGACTCGAGTCCAGGCACAGGTAATAGAT CTTTTTTG; SHCNV-NM_006674; Sigma-Aldrich; Merck $\mathrm{KGaA}$ ) or control-shRNA (CCTAAGGTTAAGTCGCCC TCGCTCGAGCGAGGGCGACTTAACCTTAGG; \#1864, Addgene, USA) were transfected into cells $(10,000)$ with Lipofectamine 2000 for $48 \mathrm{~h}$.

RNA extraction and RT-qPCR analysis. Total RNA from the tissue samples or cells was isolated using Trizol reagent
(Takara, Dalian, China). RNA $(1 \mu \mathrm{g})$ from each sample was used to synthesize the cDNA using the PrimeScript RT Master Mix (Takara, Dalian, China). Quantitative PCR was performed to detect the expression of HCP5 with the One-step SYBR PrimeScript RT-PCR kit (Takara) on the Applied Biosystem 7900 Fast Real-Time PCR System (Thermo Fisher Scientific, Inc.). The level of HCP5 was normalized to that of GAPDH. Primers used in this assay were listed as below: HCP5, forward, 5'-CCGCTGGTCTCTGGACACATACT-3' and reverse, 5'-CTCACCTGTCGTGGGATTTTGC-3'; GAPDH, forward, 5'-GTCGGTGTGAACGGATTTG-3' and reverse, 5'-AAGATGGTGATGGGCTTCC-3'. The PCR protocol was set as $95^{\circ} \mathrm{C}$ for $10 \mathrm{~min} ; 40$ cycles at $95^{\circ} \mathrm{C}$ for $10 \mathrm{sec}$ and $60^{\circ} \mathrm{C}$ for $1 \mathrm{~min}$. Relative gene expression of HCP5 was calculated using the $2^{-\Delta \Delta \mathrm{Cq}}$ method (27).

Western blot analysis. Prostate cancer cells transfected with the corresponding expression vector were harvested and lysed with RIPA buffer (Beyotime, Shanghai, China). Samples were certificated at $10,000 \mathrm{xg}$ for $10 \mathrm{~min}$ at $4^{\circ} \mathrm{C}$. The supernatant was collected and the protein concentration was quantified with the BCA kit (Bio-Rad). Protein $(20 \mu \mathrm{g})$ was separately by $15 \%$ SDS-PAGE and then transferred onto PVDF membranes (EMD Millipore). After blocking with 5\% nonfat milk, the membranes were incubated with the primary antibody against CEMIP (1:2,000 dilution; XY2112901; XYbscience, Shanghai, China), GAPDH (1:3,000 dilution; ab9485; Abcam, Shanghai, China), Snail (1:1,000 dilution; ab229701; Abcam, Shanghai, China), VEGF (1:1,000 dilution; AV202; Beyotime, Shanghai, China), E-cadherin (1:5,000 dilution; 20874-1-AP; Proteintech Group, Wuhan, China) overnight at $4^{\circ} \mathrm{C}$, followed by HRP-conjugated secondary antibody [rabbit anti-mouse IgG H\&L (HRP), 1:5,000 dilution; ab6728; Abcam; Shanghai, China; Goat anti-Rabbit IgG H\&L (HRP), 1:5,000 dilution; ab6721; Abcam; Shanghai, China] for $2 \mathrm{~h}$ at RT. The protein signal was visualized with enhanced chemiluminescence reagent (Bio-Rad).

Cell counting Kit-8 (CCK-8) assay. Cell proliferation was measured with the CCK- 8 assay (Dojindo, Tokyo, China) according to the manufacturer's instructions. Briefly, cells transfected with the corresponding expression vector were seeded in a 96-well plate in triplicate at the density of 1,000 cells per well. CCK- 8 reagent $(10 \mu \mathrm{l})$ was added into the cells at the time point of 1,2,3,4 and 5 days and incubated at $37^{\circ} \mathrm{C}$ for a further $3 \mathrm{~h}$. The absorbance of each well at $450 \mathrm{~nm}$ was measured using a microplate reader (Bio-Rad, IQ, USA).

Targes prediction. The binding between HCP5 and miR-4656 was predicted with the RNA22 version 2.0 (https://cm.jefferson. edu/rna22). The targets of miR-4656 were predicted using the miRDB database (28).

Luciferase reporter assay. To detect the binding between HCP5 and miR-4656, the wild-type (WT) or mutant (Mut) HCP5 sequences harboring the predicted binding site of miR-4656 were constructed into the pGL3 reporter vector (Promega). Similarly, to examine the binding for miR-4656 and CEMIP, the WT or Mut 3'-UTR of CEMIP was inserted into the pGL3 reporter vector. Cells cultured in a 96-well plate 
were transfected with the indicated expressing vectors with Lipofectamine 2000. After transfection for $48 \mathrm{~h}$, cells were harvested and the luciferase activity was measured using the Dual-Luciferase Reporter Assay Kit (Promega) according to the manufacturer's instructions. The assay was performed in triplicate.

Cell apoptosis. PC-3 and PPC-1 cells were transfected with shRNA-control or shRNA-HCP5 for $48 \mathrm{~h}$. The percentage of apoptotic cells was determined using the Annexin Vfluorescein-5-isothiocyanate (FITC) Apoptosis Detection kit (Invitrogen; Thermo Fisher Scientific, Inc.) according to the manufacturer's instructions. Cells were collected and washed twice with pre-cold PBS. The cell pellets were resuspended with Annexin V binding buffer. Cell resuspension (100 $\mu$ l) $(\sim 10,000$ cells) was used and stained with FITC-Annexin V and propidium iodide for $15 \mathrm{~min}$ at RT in darkness. The cell apoptosis rate was analyzed with the Becton Dickinson FACScan instrument (BD Pharmingen ${ }^{\mathrm{TM}}$, USA).

Xenograft mouse model. Both PC 3 and PPC-1 cells $\left(1 \times 10^{6}\right)$ harboring the lentivirus vector of the shRNA-control or shRNA-HCP5 were injected subcutaneously into the flank of nude mice (BALB/c, 5-6 weeks of age, female, 23-26 g; N=6 per group) and left to grow for three weeks. All mice were maintained in a specific pathogen-free (SPF) barrier condition with controlled temperature $\left(25 \pm 2^{\circ} \mathrm{C}\right)$, relative humidity $(60 \pm 5 \%)$, and 12-h light/dark cycle with free access to food and water. The tumor size was measured every three days according to the ethical IACUC guidelines. After that, mice were sacrificed by cervical dislocation and the tumor weight was measured. This experiment was approved by the Animal Research Ethics Committee of The People's Hospital of Hanchuan City. All animals were handled following the 'Guide for the Care and Use of Laboratory Animals' and the 'Principles for the Utilization and Care of Vertebrate Animals' (29).

Statistical analysis. Data are presented as mean \pm standard deviation (SD) and were analyzed with SPSS 13.0 (SPSS, Inc.). The comparison between two groups was analyzed by the Student's t-test. Comparisons among multiple groups were analyzed with ANOVA followed by a post hoc test. Spearman's rank-order correlation was applied to the correlation analysis. The r-value indicates the strength and direction of the correlation between the two variables. $\mathrm{P}<0.05$ was considered as indicative of a statistically significant difference.

\section{Results}

HCP5 is upregulated in prostate cancer tissues. To validate the expression of HCP5 in prostate cancer, the level of HCP5 was analyzed in paired prostate cancer tissues and non-tumor tissues by RT-qPCR. The result showed that HCP5 was significantly upregulated in prostate cancer tissues compared to that noted in the adjacent normal tissues (Fig. 1A). Notably, increased expression of HCP5 was positively correlated with the metastasis of prostate cancer patients (Fig. 1B). The result showed that HCP overexpression was linked to relative poor progression of prostate cancer patients. To support these data, the expression of HCP5 in prostate cancer cell lines including
PC3, Du145, PPC-1 and C4-2 and normal control cell line NHP9 was detected. As presented in Fig. 1C, overexpression of HCP was observed in all the prostate cancer cell lines when compared with the expression noted in the normal cells. These results demonstrate the high expression of HCP5 in prostate cancer.

Downregulation of HCP5 inhibits the proliferation and colony formation and induces the apoptosis of prostate cancer cells. To investigate the effect of HCP5 on the growth of prostate cancer cells, HCP5 was downregulated by transfection of shRNA-HCP5 into PPC-1 and PC3 cells. RT-qPCR assay was performed to confirm the knockdown efficiency, which showed that HCP5 expression was significantly reduced after the transfection of shRNA-HCP5 (Fig. 2A). The influence of HCP5 silencing on the growth of prostate cancer cells was evaluated with the CCK-8 assay. As indicated in Fig. 2B and C, the OD values were significantly decreased with the depletion of HCP5 in both PPC-1 and PC3 cell lines, demonstrating that knockdown of HCP5 inhibited prostate cancer cell proliferation. Consistently, the apoptotic rate (early and late apoptosis) of both PPC-1 and PC3 cell lines was significantly increased with the downregulation of HCP5 in comparison with that of the control group (Fig. 2D). Moreover, the colony formation assay was performed to evaluate the effect of HCP5 on the growth of prostate cancer cells. As presented in Fig. 2E, silencing of HCP5 significantly suppressed the colony formation ability of the PPC-1 and PC 3 cells. To further confirm the suppression of growth of prostate cancer tissues with downregulation of $\mathrm{HCP} 5$, an in vivo xenograft mouse model was established by injecting PC3 or PPC-1 cells harboring shRNA-control or shRNA-HCP5 into the flank of nude mice. The tumor weight was measured after three weeks and the result showed that depletion of HCP5 significantly decreased the tumor formation (Fig. 2F). Consistently, the tumor diameter and volumes were also decreased upon the silencing of HCP5 (Table SI). These results indicated that the downregulation of HCP5 suppressed the tumorigenesis of prostate cancer.

miR-4656 is identified as a target of HCP5. Mounting evidence suggests that lncRNAs act as competing endogenous RNA to modulate the expression of miRNAs. To further understand the function of HCP5 in regulating the growth of prostate cancer cells, the putative binding partners of HCP5 were predicted. We found that there was a complementary binding sequence to the miR-4656 seed region in HCP5 (Fig. 3A). To confirm whether HCP5 binds to miR-4656, luciferase reporter assay was performed by transfecting the luciferase vector harboring the wild-type (WT) or mutant (Mut) sequence of HCP5, which was the seeding region of miR-4656. The results showed that overexpression of miR-4656 significantly reduced the luciferase reporter activity of WT but not mutant HCP5 (Fig. 3B and C). This observation suggested the interaction between HCP5 and miR-4656 in prostate cancer cells.

To ascertain whether the binding of HCP5 to miR4656 affects the stability of miR-4656, PPC- 1 and PC 3 cells were transfected with control lncRNA or HCP5 and the level of miR-4656 was detected using the RT-qPCR assay. The data showed that ectopic expression of HCP5 significantly reduced the level of miR-4656 in both PPC-1 and PC3 cell lines (Fig. 3D). In contrast, downregulation of HCP5 

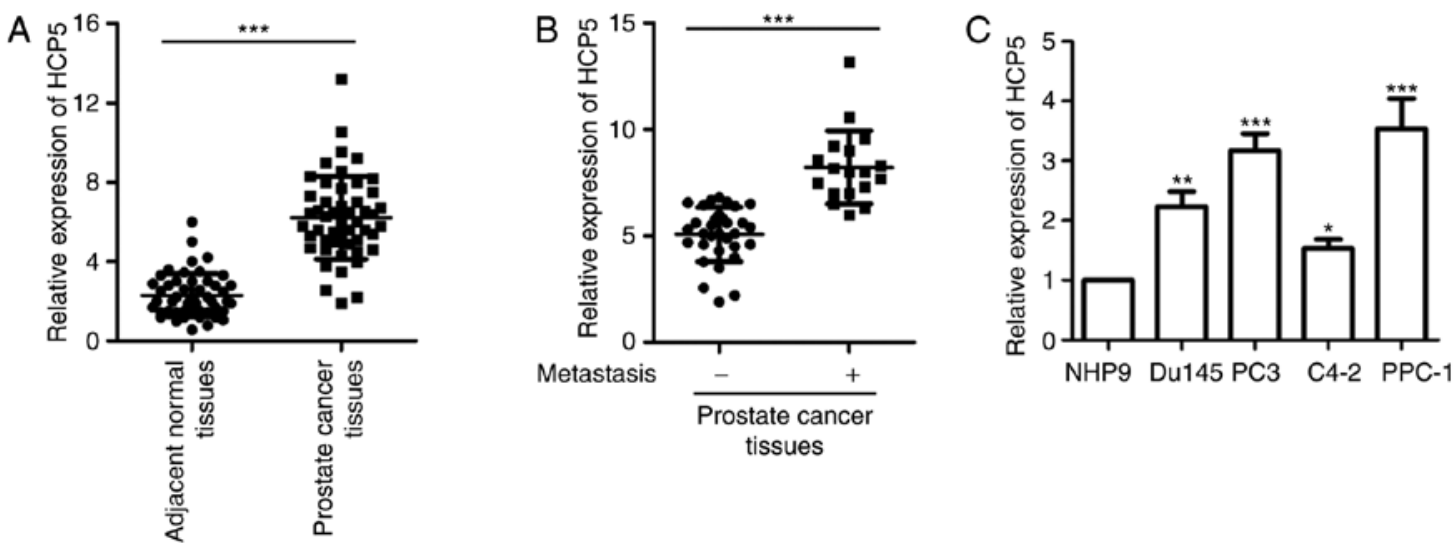

Figure 1. HCP5 is overexpressed in prostate cancer tissues. (A) The expression of HCP5 in paired prostate cancer and adjacent normal tissues was detected by RT-qPCR. (B) The expression of HCP5 was higher in prostate cancer tissues with metastasis. (C) The level of HCP5 in prostate cancer cell lines was compared with that of the normal control cell line NHP9 (vs. NHP9 cell line). HCP5, histocompatibility leukocyte antigen (HLA) complex P5. ${ }^{*}<0.05 ;{ }^{* *} \mathrm{P}<0.01$; ${ }^{* * * *} \mathrm{P}<0.001$.
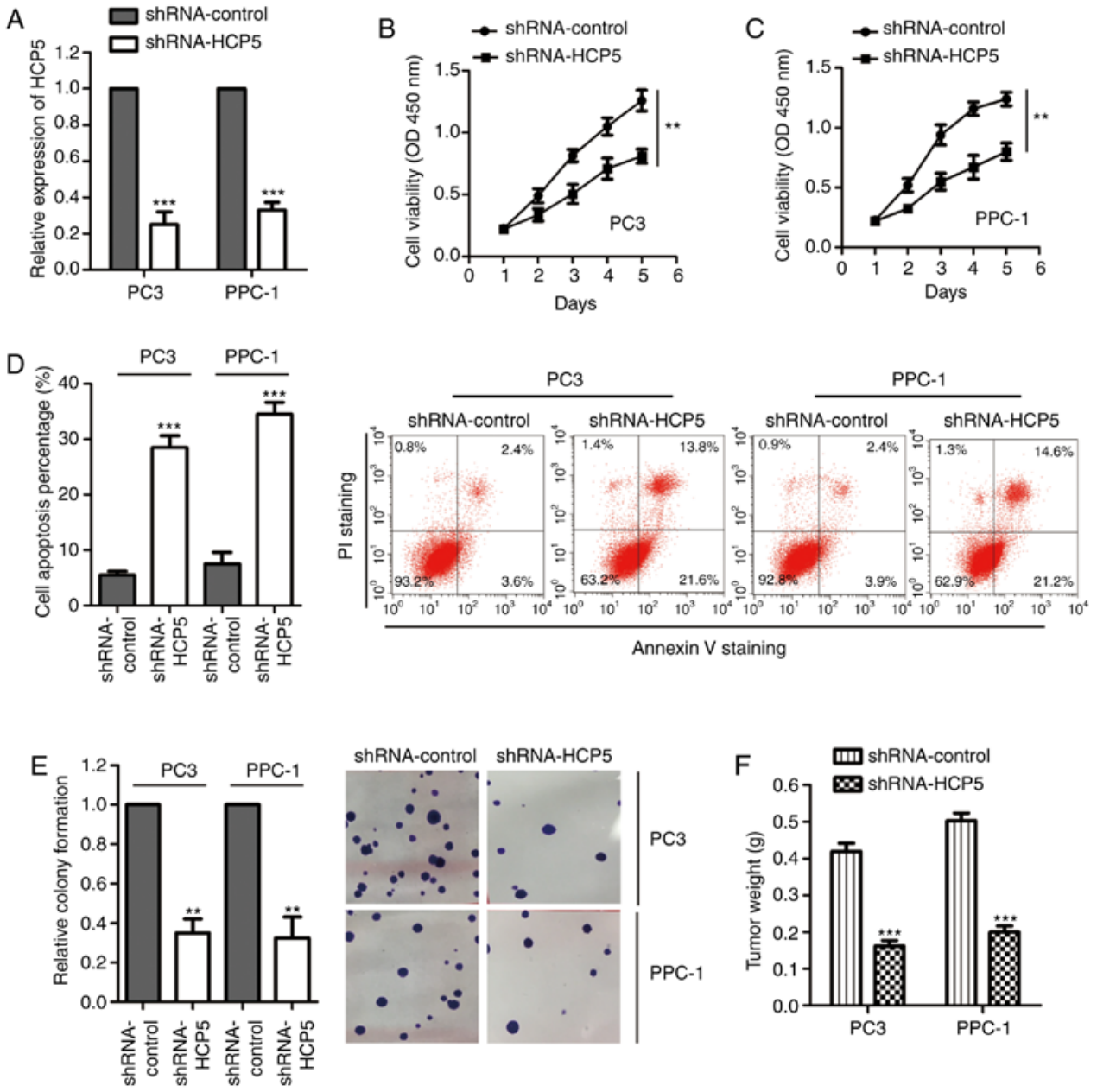

Figure 2. Downregulation of HCP5 suppresses the growth of prostate cancer cells. (A) Both PC3 and PPC-1 cell lines were transfected with the shRNA-control or shRNA-HCP5 and the downregulation of HCP5 was validated with the RT-qPCR analyses. (B and C) The proliferation of PC3 and PPC-1 cells transfected with the shRNA-control or shRNA-HCP5 was determined by the CCK-8 assay. (D) The apoptosis percentage of prostate cancer cells with downregulated HCP5 was analyzed by FACS. (E) Knockdown of HCP5 suppressed the colony formation of both PC3 and PPC-1 cells. (F) Depletion of HCP5 inhibited the growth of prostate cancer cell-derived tumors in a xenograft mouse model. HCP5, histocompatibility leukocyte antigen (HLA) complex P5. ${ }^{* *} \mathrm{P}<0.01 ;{ }^{* * *} \mathrm{P}<0.001$.

promoted the expression of miR-4656 (Fig. 3E). Supporting this observation, the expression of miR-4656 was significantly lower in prostate cancer tissues in comparison with that noted in the normal tissues (Fig. 3F), and expression of miR-4656 was inversely correlated with the level of HCP5 (Fig. 3G). These results indicate that HCP5 acts as the sponge 
A

\section{5'-UGGGCUGAGGGCAGGAGGCCUGU-3' miR-4656}

3'-TCCCGACGACCGGTATTTCTTACAA-5' HCP5

B

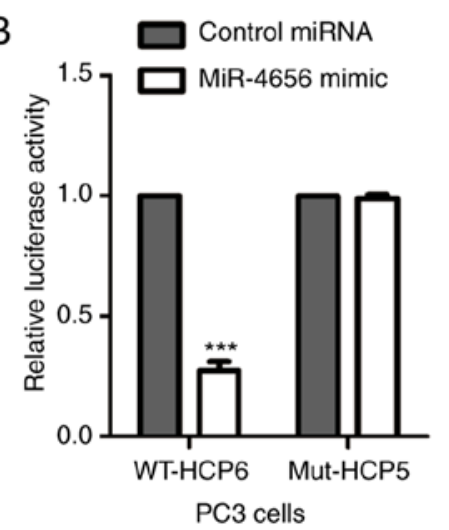

$\mathrm{D}$

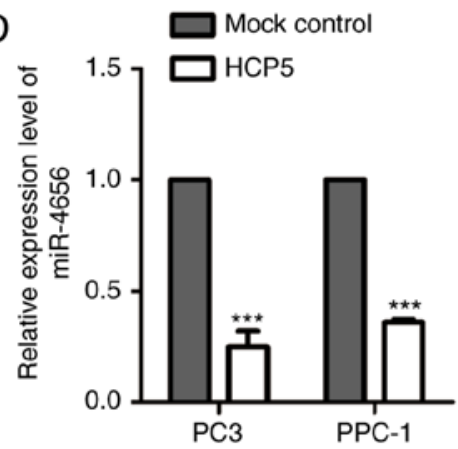

$\mathrm{F}$

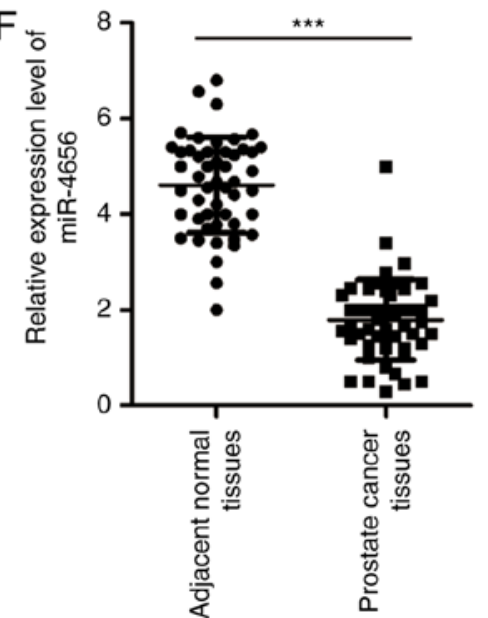

C

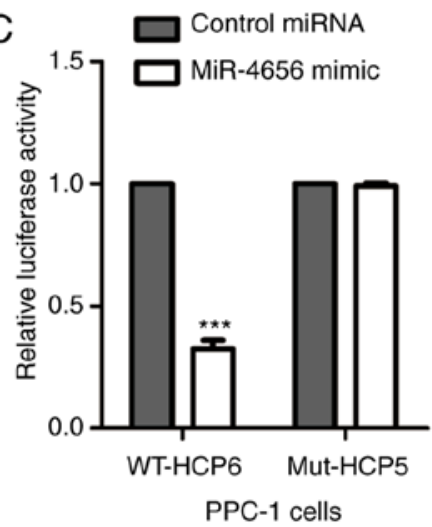

E

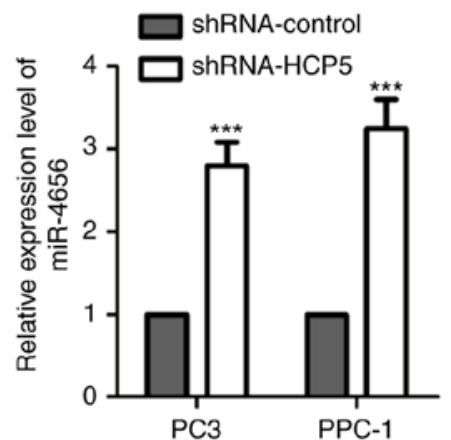

G

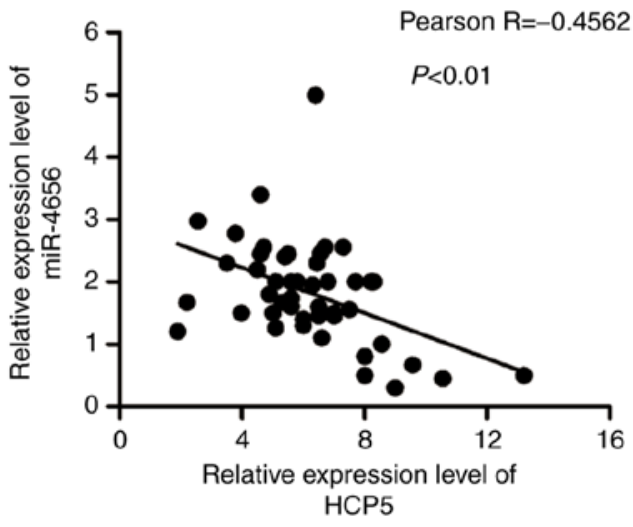

Figure 3. HCP5 is a sponge of miR-4656 in prostate cancer cells. (A) The predicted binding sites of HCP5 within the sequence of miR-4656. (B and C) Overexpression of miR-4656 decreased the luciferase activity of wild-type (WT) but not the mutant (Mut) HCP5. (D and E) Overexpression of HCP5 inhibited the expression of miR-4656 in both PC3 and PPC-1 cells, while depletion of HCP5 increased the level of miR-4656. (F) The expression of miR-4656 in paired prostate cancer tissues and adjacent normal tissues was detected with RT-qPCR assay. (G) The correlation between the expression of HCP5 and miR-4656 was examined with the Spearman's test. HCP5, histocompatibility leukocyte antigen (HLA) complex P5. ${ }^{* * *} \mathrm{P}<0.001$.

of miR-4656 and reduced the expression of miR-4656 in prostate cancer cells.

miR-4656 targets CEMIP in prostate cancer cells. To detect the effect of miR-4656 on the growth of prostate cancer cells, both PC-3 and PPC-1 cell lines were transfected with
miRNA control or miR-4656 mimic to upregulate the expression of miR-4656 (Fig. 4A). The CCK-8 assay showed that overexpression of miR-4656 significantly decreased the proliferation of both PC-3 and PPC-1 cell lines (Fig. 4B and C). To further understand the molecular mechanisms by which miR-4656 regulates the proliferation of prostate cancer cells, 

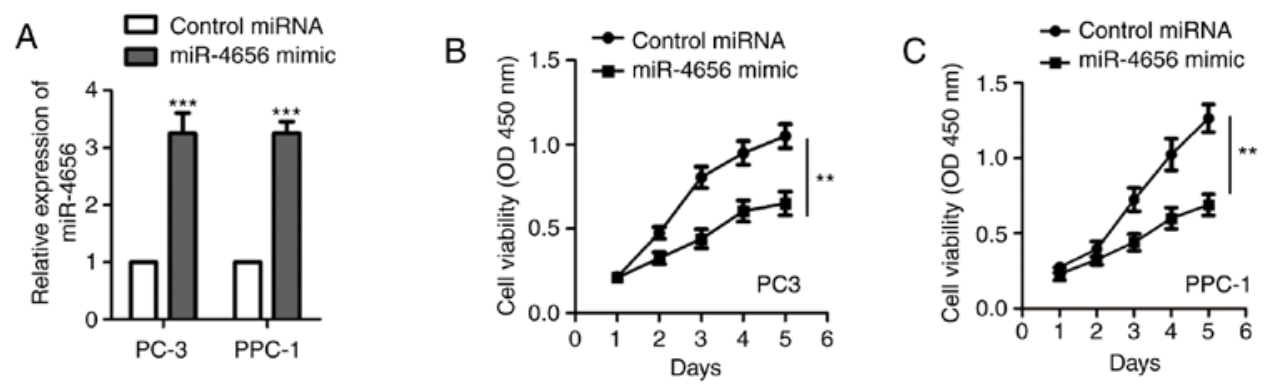

\section{5 -UGGGCUGAGGGCAGGAGGCCUGU-3 miR-4656 |||| $\mid$ \\ 3 -TCCCGACTTTGTGCCGGAAGGGTCC-5 3 -UTR of CEMIP}
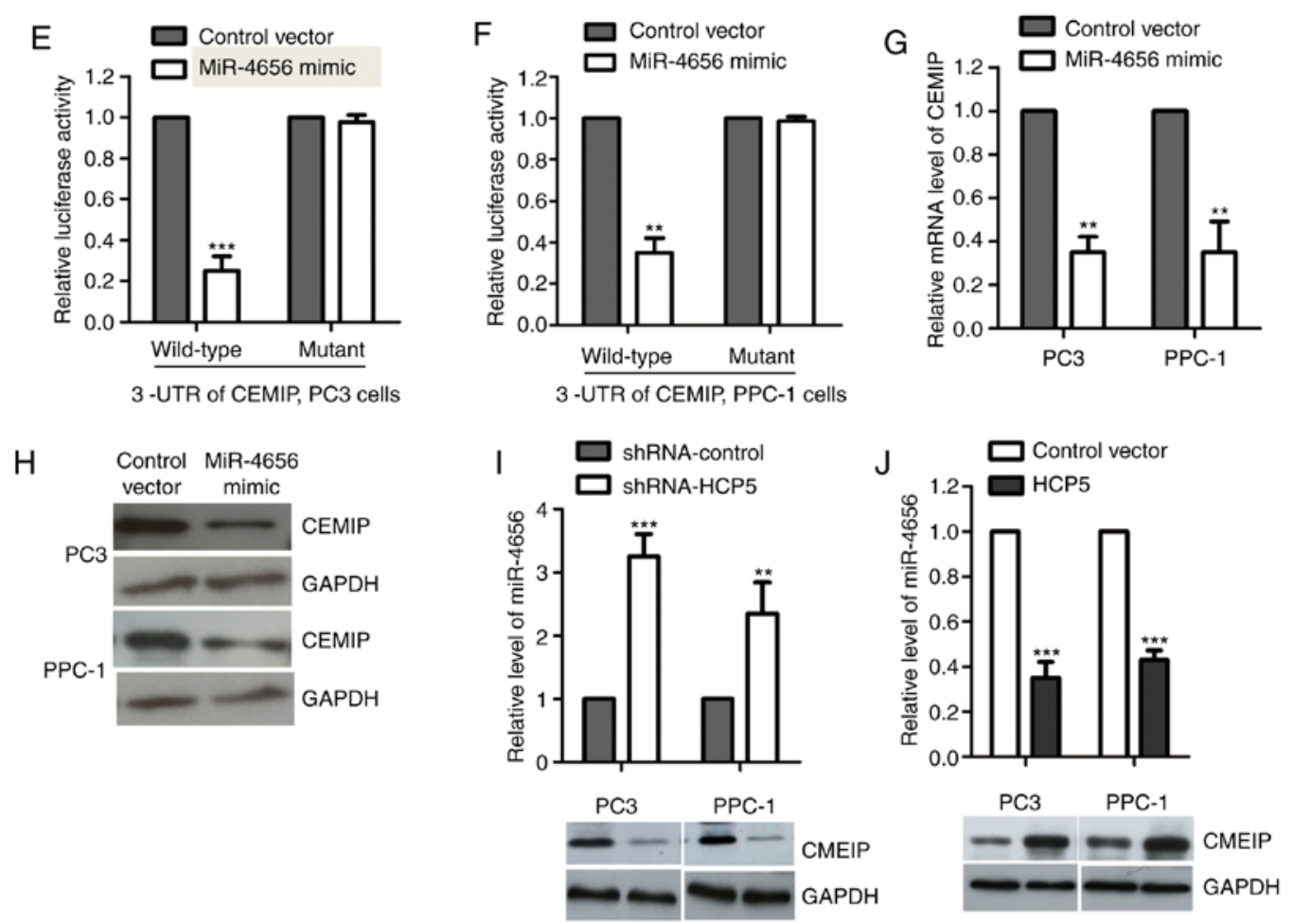

Figure 4. miR-4656 targets CEMIP in prostate cancer cells. (A) PC-3 and PPC-1 cells were transfected with control miRNA or miR-4656 mimic and the expression of miR-4656 was confirmed by RT-qPCR. (B and C) Overexpression of miR-4656 inhibited the proliferation of prostate cancer cells. (D) The predicted binding sites of miR-4656 at the 3'-UTR of CEMIP. (E and F) PC-3 and PPC-1 cells were transfected with control vector or miR-4656 mimic and the luciferase activity was measured. (G and H) High expression of miR-4656 decreased the mRNA and protein levels of CEMIP in both PC3 and PPC-1 cells. (I and J) Cells were transfected with shRNA-HCP5 or an overexpressing HCP vector, and the levels of miR-4656 and CEMIP were detected. ** P $<0.01$; ${ }_{* * * *} \mathrm{P}<0.001$. CEMIP, cell migration inducing hyaluronidase 1; HCP5, histocompatibility leukocyte antigen (HLA) complex P5.

the possible targets of miR-4656 were predicted using the miRDB database. The prediction revealed that CEMIP is a potential binding candidate of miR-4656 (Fig. 4D). Increasing evidence has demonstrated the oncogenic function of CEMIP in promoting tumorigenesis by modulating cancer-related signaling pathways (30-33). The overexpression of CEMIP in tumors provides a novel target for individualized therapy (34). However, the involvement of CEMIP in the malignancy of prostate cancer has not been fully understood. There were three target sites of miR-4656 in the 3'-UTR of CEMIP that were predicted and the first one (seed location 121) was examined for further analysis. To confirm this, luciferase reporter assay was performed by constructing the WT or mutant 3'-UTR which contained the binding sites of miR-4656 into the luciferase reporter vector. The results showed that overexpression of miR-4656 significantly reduced the WT but not the mutant 3'-UTR luciferase activity (Fig. 4E and F). RT-qPCR assay showed that high expression of miR-4656 decreased both the mRNA and protein levels of CEMIP in the PPC-1 and PC3 cell lines (Fig. 4G and $\mathrm{H}$ ). To check the co-expression of HCP5/miR-4656/CEMIP, cells were transfected with HCP-5 or shRNA-HCP5. The results showed that depletion of HCP5 significantly upregulated the level of miR-4656 and decreased the expression of CEMIP (Fig. 4I). Consistently, overexpression of HCP5 significantly suppressed miR-4656 and increased the abundance of CEMIP (Fig. 4J). Our results identified CEMIP as a target of the HCP5/miR-4656 axis in prostate cancer cells.

HCP5 regulates the miR-4656/CEMIP pathway. To determine whether HCP5 regulates CEMIP via suppression of miR-4656, 
A

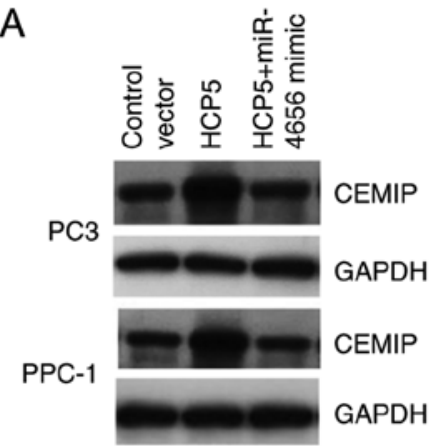

B

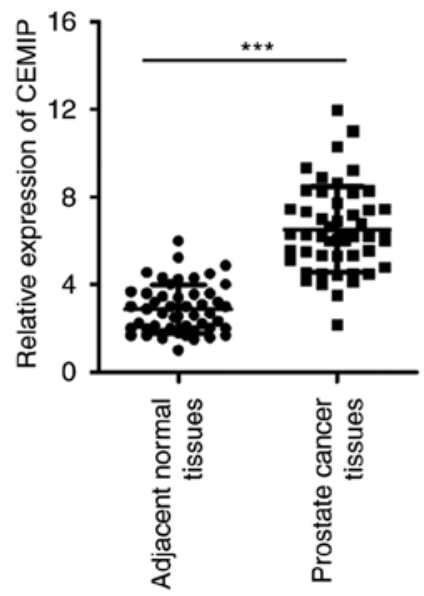

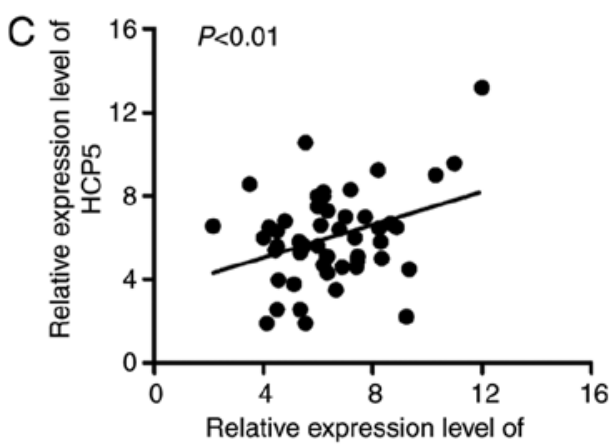

CEMIP
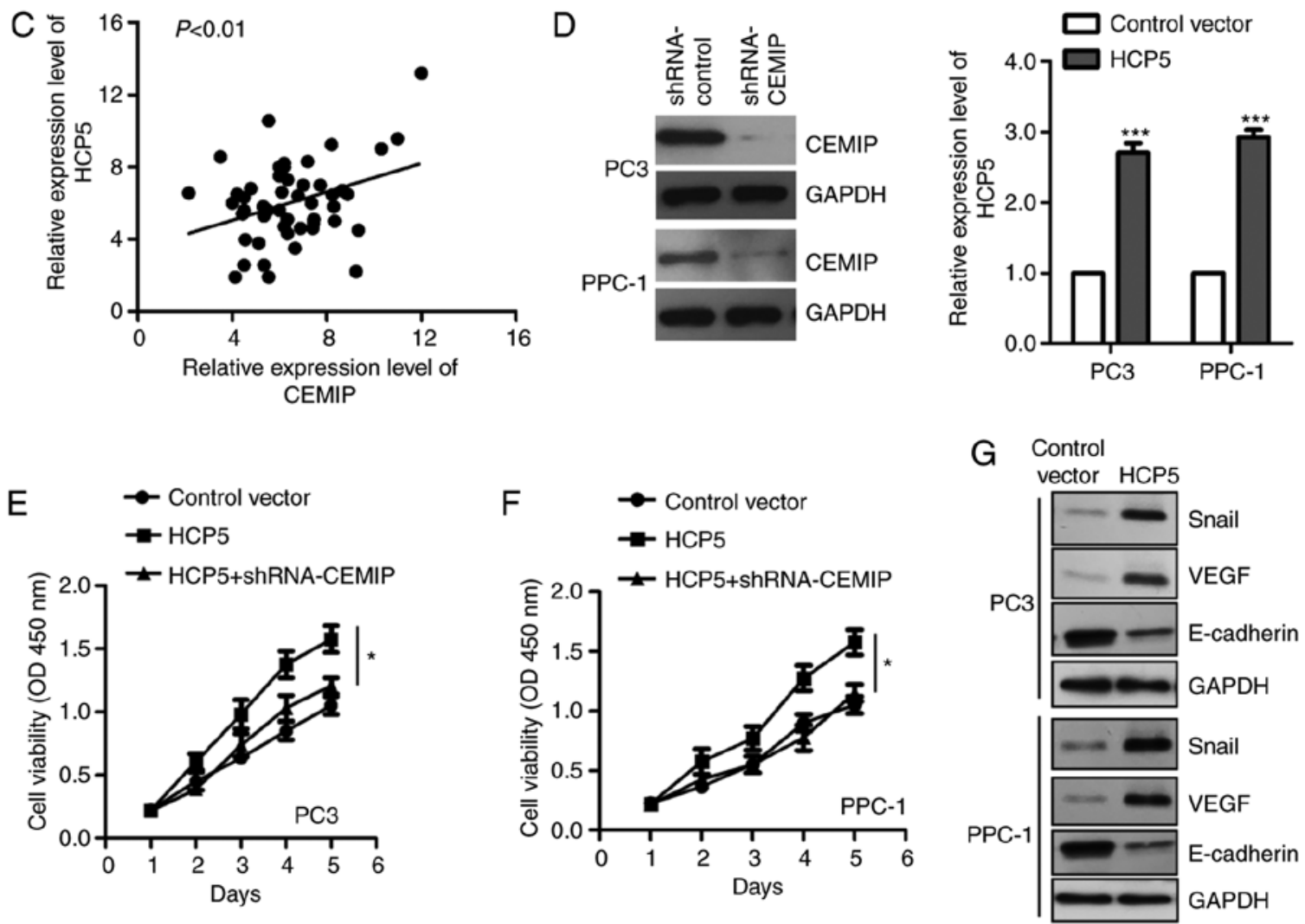

Figure 5. HCP5 upregulates the expression of CEMIP in prostate cancer cells. (A) In the PC3 and PPC-1 cells overexpressing miR-4656 and HCP5, the protein level of CEMIP was detected. (B) The expression of CEMIP in paired prostate cancer and adjacent normal tissues was detected by RT-qPCR. (C) The correlation between the level of HCP5 and CEMIP was determined with Spearman's test. (D) Transfection of shRNA-CEMIP decreased the expression of CEMIP in prostate cancer cells. The level of HCP5 was significantly increased following the transfection of HCP5. (E and F) PC3 and PPC-1 cells were transfected with the indicated expression vectors and the cell proliferation was determined by the CCK-8 assay. (G) Both PC3 and PPC-1 cells were transfected with control-shRNA or HCP5, and the level of Snail, VEGF and E-cadherin were determined by western blot analysis. " $\mathrm{P}<0.05$; ${ }^{* * *} \mathrm{P}<0.001$. CEMIP, cell migration inducing hyaluronidase 1; HCP5, histocompatibility leukocyte antigen (HLA) complex P5.

miR-4656 was overexpressed and the expression of CEMIP was detected. The data showed that rescue of miR-4656 reversed the upregulation of CEMIP by HCP5 (Fig. 5A). To further support the correlation between HCP5 and CEMIP, the mRNA level of CEMIP in paired prostate cancer tissues and adjacent normal tissues was compared via RT-qPCR. The data revealed significantly increased expression of CEMIP in the prostate cancer tissues (Fig. 5B). Meanwhile, the correlation between the abundance of HCP5 and CEMIP was analyzed by Spearman test. As indicated in Fig. 5C, the expression of CEMIP was positively correlated with that of HCP5 in prostate cancer tissues. To verify whether CEMIP mediates the function of HCP5 in prostate cancer, CEMIP was downregulated and HCP5 was overexpressed in both PC-3 and PPC-1 cell lines (Fig. 5D). The CCK-8 assay showed that knockdown of CEMIP reduced the HCP5-promoted proliferation of PC-3 and PPC-1 cells (Fig. 5E and F). These data indicate that CEMIP is regulated by HCP5 and modulates the growth of prostate 
cancer cells. Increasing studies have demonstrated that CEMIP orchestrates tumorigenesis via regulating $\mathrm{Wnt} / \beta$-catenin signaling (33). To further demonstrate the functional mechanism of CEMIP in prostate cancer, we evaluated the expression of several targets of the Wnt/ $\beta$-catenin pathway including Snail, E-cadherin, and VEGF. The results showed that overexpression of HCP5 upregulated Snail and VEGF, and inhibited the level of E-cadherin in both PC3 and PPC1 cell lines (Fig. 5G). These data provide the possible mechanism underlying the regulatory function of CEMIP in prostate cancer.

\section{Discussion}

Accumulating evidence indicates the important roles of long noncoding RNAs (lncRNAs) in the initiation and development of human cancers $(9,35)$. Considering the high occurrence of prostate cancer in the male population and the high mortality rate of prostate cancer patients, understanding the molecular mechanism of lncRNAs in regulating the growth of prostate cancer cells may provide new therapeutic strategies for the treatment of prostate cancer. In the present study, we identified that lncRNA histocompatibility leukocyte antigen (HLA) complex P5 (HCP5) is overexpressed in prostate cancer tissues and cell lines. Mechanistically, HCP5 was found to promote the proliferation of prostate cancer cells via sponging miR-4656 to upregulate the expression of CEMIP.

HCP5 is located on chromosome 6p21.3, and the function of HCP5 is not fully understood (36). A recent study demonstrated that HCP5 is overexpressed in glioma tissues and is positively correlated with the histopathological grade of glioma cancer patients (25). Knockdown of HCP5 was found to inhibit the malignancy of glioma cells by upregulating miR-139. These data suggest the oncogenic function of HCP5 in glioma. Increased expression of HCP5 was also identified in follicular thyroid carcinoma (FTC). HCP5 was found to promote the tumorigenesis of FTC (37). Mechanistic experiments indicated that HCP5 functions as a ceRNA to sponge miR-22-3p, miR-186-5p and miR-216-5p, which triggers activity of alpha-2, 6-siallyltransferase 2 in FTC cells (37). Consistently, our results showed the upregulation of the expression of HCP5 in prostate cancer, which was associated with the worse prognosis of prostate cancer patients. Functional experiments showed that downregulation of HCP5 suppressed the proliferation and induced the apoptosis of prostate cancer cell lines. To further explore the molecular mechanism by which HCP5 regulates the tumorigenesis of prostate cancer, the miRNA targets of HCP5 were predicted with the miRDB database. The results identified miR-4656 as a potential target of HCP5. Overexpression of HCP5 significantly decreased the abundance of miR-4656 in prostate cancer cells. Our data uncovered the critical involvement of the HCP5/miR-4656 axis in prostate cancer.

The function of miRNAs in regulating the growth of cancer cells occurs mainly by inhibiting the expression of downstream target genes. The involvement of miR-4656 in cancer is not fully understood. Recent research showed that miR-4656 is aberrantly expressed in glioma tissues and is correlated with the prognosis of glioma cancer patients (38). In the present study, to further understand the functional mechanism of miR-4656 in prostate cancer, downstream targets of miR-4656 were predicted. The data showed that miR-4656 bound the
3'-UTR of CEMIP and suppressed the expression of CEMIP. CEMIP was primary identified as a hearing loss-related gene (39). Interestingly, increased expression of CEMIP has been identified in a variety of human cancer (31,32,39-41). For example, upregulated expression of CEMIP may be a valuable biomarker for the detection of pancreatic cancer at an early stage (39). A recent study showed that overexpression of CEMIP is triggered by the AMPK/GSK $3 \beta / \beta$-catenin cascade and promotes the invasion and migration of prostate cancer cells via elevating metabolic reprogramming. These results demonstrated that the targeting of CEMIP may be a promising strategy for the treatment of advanced prostate cancer patients (42). The oncogenic function of CEMIP was also found in colorectal cancer $(31,40,41)$. In this study, overexpression of HCP5 enhanced the abundance of CEMIP. These results provided the possible mechanism by which HCP5 regulates the growth of prostate cancer cells.

In conclusion, our data revealed that HCP5 is overexpressed in prostate cancer tissues, and is correlated with a worse prognosis of cancer patients. For further study, immunostaining of prostate cancer tissues is necessary to evaluate the cancer grade and neovascularization using fresh samples. Functional mechanistic investigation uncovered that HCP5 promotes the growth of prostate cancer cells via acting as the sponge of miR-4656 and consequently upregulated the expression of CEMIP. HCP5 may be a potential target for designing a novel therapeutic strategy for prostate cancer.

\section{Acknowledgements}

Not applicable.

\section{Funding}

No funding was received.

\section{Availability of data and materials}

The datasets used during the present study are available from the corresponding authors upon reasonable request.

\section{Authors' contributions}

$\mathrm{RH}$ and $\mathrm{ZL}$ designed the experiments. RH performed the experiments. RH and ZL analyzed the data and wrote the manuscript.

\section{Ethics approval and consent to participate}

The study was approved by the Ethics Committee of The People's Hospital of Hanchuan City (accession no. LL2017014). Informed consents were received from all the patients.

\section{Patient consent for publication}

Not applicable.

\section{Competing interests}

The authors declare that they have no competing interests. 


\section{References}

1. Jemal A, Bray F, Center MM, Ferlay J, Ward E and Forman D: Global cancer statistics. CA Cancer J Clin 61: 69-90, 2011.

2. Yamamoto S, Kawakami S, Yonese J, Fujii Y, Urakami S, Masuda H, Numao N, Ishikawa Y, Kohno A and Fukui I: Long-term oncological outcome and risk stratification in men with high-risk prostate cancer treated with radical prostatectomy. Jpn J Clin Oncol 42: 541-547, 2012.

3. Hoang DT, Iczkowski KA, Kilari D, See W and Nevalainen MT: Androgen receptor-dependent and -independent mechanisms driving prostate cancer progression: Opportunities for therapeutic targeting from multiple angles. Oncotarget 8: 3724-3745, 2017.

4. Jeong CW, Kang M, Il Jung S, Kim TH, Park SW, Joung JY, Jeon SS, Hong JH, Lee JY, Chung BH, et al: Importance of androgen-deprivation therapy during enzalutamide treatment in men with metastatic castration-resistant prostate cancer following chemotherapy: Results from retrospective, multicenter data. Prostate Cancer Prostatic Dis 22: 150-158, 2019.

5. Onozawa M, Akaza H, Hinotsu S, Oya M, Ogawa O, Kitamura T, Suzuki K, Naito S, Namiki M, Nishimura K, et al: Combined androgen blockade achieved better oncological outcome in androgen deprivation therapy for prostate cancer: Analysis of community-based multi-institutional database across Japan using propensity score matching. Cancer Med 7: 4893-4902, 2018.

6. Siddiqui ZA and Krauss DJ: Adjuvant androgen deprivation therapy for prostate cancer treated with radiation therapy. Trans Androl Urol 7: 378-389, 2018.

7. Wu Z, Liu X, Liu L, Deng H, Zhang J, Xu Q, Cen B and Ji A: Regulation of lncRNA expression. Cell Mol Biol Lett 19: 561-575, 2014.

8. Khorkova O, Hsiao J and Wahlestedt C: Basic biology and therapeutic implications of lncRNA. Adv Drug Deliv Rev 87: 15-24, 2015.

9. Balas MM and Johnson AM: Exploring the mechanisms behind long noncoding RNAs and cancer. Noncoding RNA Res 3: 108-117, 2018.

10. Yang S, Sun Z, Zhou Q, Wang W, Wang G, Song J, Li Z, Zhang Z, Chang Y, Xia K, et al: MicroRNAs, long noncoding RNAs, and circular RNAs: Potential tumor biomarkers and targets for colorectal cancer. Cancer Manag Res 10: 2249-2257, 2018.

11. Olgun G, Sahin O and Tastan O: Discovering lncRNA mediated sponge interactions in breast cancer molecular subtypes. BMC Genomics 19: 650, 2018.

12. Paraskevopoulou MD and Hatzigeorgiou AG: Analyzing MiRNA-LncRNA Interactions. Methods Mol Biol 1402: 271-286, 2016.

13. Bartel DP: MicroRNAs: Genomics, biogenesis, mechanism, and function. Cell 116: 281-297, 2004.

14. Mohr AM and Mott JL: Overview of microRNA biology. Semin Liver Dis 35: 3-11, 2015.

15. Ambros V: The functions of animal microRNAs. Nature 431 350-355, 2004

16. Fabian MR, Sonenberg N and Filipowicz W: Regulation of mRNA translation and stability by microRNAs. Annu Rev Biochem 79: 351-379, 2010 .

17. Ye S, Yang L, Zhao X, Song W, Wang W and Zheng S: Bioinformatics method to predict two regulation mechanism: TF-miRNA-mRNA and lncRNA-miRNA-mRNA in pancreatic cancer. Cell Biochem Biophys 70: 1849-1858, 2014.

18. Hao NB, He YF, Li XQ, Wang K and Wang RL: The role of miRNA and lncRNA in gastric cancer. Oncotarget 8: 81572-81582, 2017.

19. Zhang G, Pian C, Chen Z, Zhang J, Xu M, Zhang L and Chen Y: Identification of cancer-related miRNA-lncRNA biomarkers using a basic miRNA-IncRNA network. PLoS One 13. e0196681, 2018.

20. Huang T, Liu HW, Chen JQ, Wang SH, Hao LQ, Liu M and Wang B: The long noncoding RNA PVT1 functions as a competing endogenous RNA by sponging miR-186 in gastric cancer. Biomed Pharmacother 88: 302-308, 2017.

21. Yang B, Gao G, Wang Z, Sun D, Wei X, Ma Y and Ding Y: Long non-coding RNA HOTTIP promotes prostate cancer cells proliferation and migration by sponging miR-216a-5p. Biosci Rep 38: pii: BSR20180566, 2018.

22. Tian C, Deng Y, Jin Y, Shi S and Bi H: Long non-coding RNA RNCR3 promotes prostate cancer progression through targeting miR-185-5p. Am J Transl Res 10: 1562-1570, 2018.
23. Liu Y, Helms C, Liao W, Zaba LC, Duan S, Gardner J, Wise C, Miner A, Malloy MJ, Pullinger CR, et al: A genome-wide association study of psoriasis and psoriatic arthritis identifies new disease loci. PLoS Genet 4: e1000041, 2008.

24. Yu Y, Shen HM, Fang DM, Meng QJ and Xin YH: LncRNA HCP5 promotes the development of cervical cancer by regulating MACC1 via suppression of microRNA-15a. Eur Rev Med Pharmacol Sci 22: 4812-4819, 2018.

25. Teng H, Wang P, Xue Y, Liu X, Ma J, Cai H, Xi Z, Li Z and Liu Y: Role of HCP5-miR-139-RUNX1 feedback loop in regulating malignant behavior of glioma cells. Mol Ther 24: 1806-1822, 2016.

26. Lange CM, Bibert S, Dufour JF, Cellerai C, Cerny A, Heim MH, Kaiser L, Malinverni R, Müllhaupt B, Negro F, et al: Comparative genetic analyses point to HCP5 as susceptibility locus for HCV-associated hepatocellular carcinoma. J Hepatol 59: 504-509, 2013.

27. Livak KJ and Schmittgen TD: Analysis of relative gene expression data using real-time quantitative PCR and the 2(-Delta Delta $\mathrm{C}(\mathrm{T}))$ method. Methods 25: 402-408, 2001.

28. Wong N and Wang X: miRDB: An online resource for microRNA target prediction and functional annotations. Nucleic Acids Res 43 (Database Issue): D146-D152, 2015.

29. U.S. National Institutes of Health: Laboratory animal welfare; proposed U.S. government principles for the utilization and care of vertebrate animals used in testing, research and training. Fed Regist 49: 29350-29351, 1984.

30. Shen F, Zong ZH, Liu Y, Chen S, Sheng XJ and Zhao Y: CEMIP promotes ovarian cancer development and progression via the PI3K/AKT signaling pathway. Biomed Pharmacother 114: 108787, 2019

31. Fink SP, Myeroff LL, Kariv R, Platzer P, Xin B, Mikkola D, Lawrence E, Morris N, Nosrati A, Willson JK, et al: Induction of KIAA1199/CEMIP is associated with colon cancer phenotype and poor patient survival. Oncotarget 6: 30500-30515, 2015.

32. Liang G, Fang X, Yang Y and Song Y: Knockdown of CEMIP suppresses proliferation and induces apoptosis in colorectal cancer cells: Downregulation of GRP78 and attenuation of unfolded protein response. Biochem Cell Biol 96: 332-341, 2018.

33. Liang G, Fang X, Yang Y and Song Y: Silencing of CEMIP suppresses Wnt/ $\beta$-catenin/Snail signaling transduction and inhibits EMT program of colorectal cancer cells. Acta Histochem 120: 56-63, 2018

34. Li L, Yan LH, Manoj S, Li Y and Lu L: Central role of CEMIP in tumorigenesis and its potential as therapeutic target. J Cancer 8: 2238-2246, 2017.

35. Corra F, Agnoletto C, Minotti L, Baldassari F and Volinia S: The network of Non-coding RNAs in cancer drug resistance. Front Oncol 8: 327, 2018

36. Medici M, Porcu E, Pistis G, Teumer A, Brown SJ, Jensen RA, Rawal R, Roef GL, Plantinga TS, Vermeulen SH, et al: Identification of novel genetic Loci associated with thyroid peroxidase antibodies and clinical thyroid disease. PLoS Genet 10: e1004123, 2014

37. Liang L, Xu J, Wang M, Xu G, Zhang N, Wang G and Zhao Y: LncRNA HCP5 promotes follicular thyroid carcinoma progression via miRNAs sponge. Cell Death Dis 9: 372, 2018.

38. Shou J, Gu S and Gu W: Identification of dysregulated miRNAs and their regulatory signature in glioma patients using the partial least squares method. Exp Ther Med 9: 167-171, 2015.

39. Lee HS, Jang CY, Kim SA, Park SB, Jung DE, Kim BO, Kim HY, Chung MJ, Park JY, Bang S, et al: Combined use of CEMIP and CA 19-9 enhances diagnostic accuracy for pancreatic cancer. Sci Rep 8: 3383, 2018.

40. Zhang D, Zhao L, Shen Q, Lv Q, Jin M, Ma H, Nie X, Zheng X, Huang S, Zhou P, et al: Down-regulation of KIAA1199/CEMIP by miR-216a suppresses tumor invasion and metastasis in colorectal cancer. Int J Cancer 140: 2298-2309, 2017.

41. Evensen NA, Li Y, Kuscu C, Liu J, Cathcart J, Banach A, Zhang Q, Li E, Joshi S, Yang J, et al: Hypoxia promotes colon cancer dissemination through up-regulation of cell migration-inducing protein (CEMIP). Oncotarget 6: 20723-20739, 2015.

42. Zhang P, Song Y, Sun Y, Li X, Chen L, Yang L and Xing Y: AMPK/GSK3 $/ / \beta$-catenin cascade-triggered overexpression of CEMIP promotes migration and invasion in anoikis-resistant prostate cancer cells by enhancing metabolic reprogramming. FASEB J 32: 3924-3935, 2018. 